Ann. Génét. Sél. anim., I973, 5 (4), 425-434.

\title{
BANDES CHROMOSOMIQUES DE TYPE G GHEZ LE LAPIN DOMESTIQUE (ORYCTOLAGUS CUNICULUS)
}

\author{
Geneviève ECHARD \\ Laboratoive de Génétique cellulaire, \\ Centre de Recherches de Toulouse, I. N. R. A., \\ B. P. 12, 31320 Castanet Tolosan
}

RÉSUMÉ

Les techniques permettant d'obtenir les bandes spécifiques des chromosomes d'une espèce donnée, sont adaptées à des cultures de moelle osseuse de lapin. Les cellules métaphasiques, étalées sur lames ont subi un prétraitement à la trypsine avant d'être colorées au Giemsa. Les caryotypes réalisés ont permis de déterminer la répartition des bandes des 2 I paires d'autosomes et d'identifier les chromosomes $\mathrm{X}$.

\section{INTRODUCTION}

Les techniques d'étude du caryotype mises au point au cours de ces dernières années et permettant d'identifier chaque chromosome par ses bandes spécifiques, constituent maintenant un outil indispensable en cytogénétique, en particulier pour caractériser les chromosomes sexuels et pour l'étude des anomalies chromosomiques pathologiques. L'étude des anomalies chromosomiques à l'aide des techniques de bandes, actuellement très engagée chez 1'Homme, serait intéressante à entreprendre dans le cas des espèces animales zootechniques. Elle nécessite, en première étape une connaissance précise des bandes chromosomiques de chaque paire d'homologues de l'espèce considérée. Le caryotype à bandes des espèces animales zootechniques commence à être étudié : bovins (HANSEN, I972-I973), porcins (GuSTAvsSON, HAGELTORN et al., I972; HANSEN, I972; BERGER, I972; ECHARD, I973), ovins (NADler, HofFMann et al., I973), caprins (HANSEN, I973). Le Lapin étant une espèce 
à large diffusion zootechnique, faisant l'objet de nombreux travaux de génétique, nous avons étudié son caryotype à l'aide des techniques de bandes. La connaissance des bandes chromosomiques facilitera ultérieurement les recherches sur la nature et l'origine des anomalies chromosomiques en particulier celles dont la présence et 1a fréquence entraînent une mortalité notable au cours de l'embryogenèse (HoFSAEss, MEACHAM, I969; FEChHEIMER, I972), ou une baisse de viabilité des spermatozoïdes.

Le caryotype du Lapin comporte 44 chromosomes (2I paires d'autosomes et une paire de chromosomes sexuels). Ce chiffre a été établi par PAINTER en I926 et confirmé ensuite par différents chercheurs parmi lesquels MELANDER (I956). A partir de I960, le développement des techniques de culture des leucocytes du sang ayant facilité les travaux de cytogénétique, de nombreux auteurs ont cherché à répartir en groupes les 22 paires de chromosomes du Lapin. Les critères utilisés ont été la longueur et la position du centromère (GUSTAVsson, I964; DAVE et TAKAGI, I965), auxquels viennent s'ajouter des mesures biométriques ou des études autoradiographiques (Nichols, LEVAN et al., I965; Willitams et RAy, I966, ISSA, ATHERTON et al., I968). Il n'y a pas actuellement de nomenclature standardisée en ce qui concerne le caryotype du Lapin. La classification adoptée est celle donnée par Gustavsson en I964.

\section{MATÉRIEL, E'T TECHNIQUES}

\section{1. - Obtention des lames}

Les cellules métaphasiques ont été obtenues en utilisant les techniques classiques de culture de moelle osseuse déjà utilisées chez le Lapin (Nichols, Levan et al., I965). De légères modifications ont été apportées à cette technique, ses modalités sont les suivantes :

Les fémurs de lapins sont prélevés juste après l'abattage. La moelle osseuse retirée au niveau de la tête du fémur, est dissociée dans des tubes coniques contenant chacun $5 \mathrm{ml}$ de milieu 199 complémenté avec $4 \mathrm{p}$. Ioo de sérum de veau foetal et auquel est ajouté de la colchicine à la concentration finale de $0,4 \mu \mathrm{g} / \mathrm{ml}$. Les tubes sont ensuite incubés I h à I h $30 \mathrm{mn}$ à $37^{\circ}$. Deux types de milieux hypotoniques sont utilisés, soit une solution de citrate de sodium à I p. Ioo, soit une solution de KCL à 0,7 p. IOo. Dans les deux cas, la durée du choc est de 40 minutes à $37^{\circ} \mathrm{C}$.

Les cellules sont ensuite fixées à l'aide d'un Carnoy sans chloroforme, étalées sur lames et séchées à l'air.

\section{2. - Prétraitement et coloration}

La technique utilisée en vue de l'obtention des bandes fait intervenir un prétraitement à la trypsine suivi d'une coloration au Giemsa (T-G-Technique). Elle dérive de la technique établie par SEABRIGHT, I97 I, puis WANG et FEDOROFF, I972; elle a été appliquée comme suit à la moelle osseuse du Lapin :

Les lames sont plongées pendant deux à trois minutes, à température ambiante, dans une solution de trypsine à o,025 p. Ioo (trypsine diluée dans du $\mathrm{NaCl}$ o, 8 p. I00). Les lames sont ensuite rinçées à l'eau distillée, plongées dans le tampon phosphate utilisé pour diluer le Giemsa, colorées 5 minutes au Giemsa, puis différenciées dans l'eau distillée.

Cette technique par digestion enzymatique à la trypsine permet d'obtenir les bandes de type G (Conférence de Paris, r97r). Ces bandes sont aisément reproductibles, mais il suffit d'un traitement un peu trop poussé pour obtenir des chromosomes à aspect dilacéré et dont le contour n'est plus nettement reconnaissable. Cet inconvénient a déjà été rencontré dans le cas d'une digestion à la trypsine (LEE, WELCH et al., 1972). 


\section{RÉSULTATS}

Les chromosomes du lapin sont répartis en six groupes (A-B-C-D-E-F) d'après leur longueur ( $\mathrm{I}^{\mathrm{er}}$ critère) puis la position de leur centromère $\left(2^{\mathrm{e}}\right.$ critère). A l'intérieur de chaque groupe, ils sont disposés par taille décroissante et un numéro est affecté à chaque paire. Les groupes $\mathrm{A}, \mathrm{B}, \mathrm{C}, \mathrm{E}, \mathrm{F}$ contiennent 4 paires de chromosomes, seul le groupe $\mathrm{D}$ n'en comprend que deux. Les groupes $\mathrm{A}, \mathrm{C}, \mathrm{E}$ sont formés de chromosomes métacentriques et submétacentriques, les groupes $\mathrm{B}$ et $\mathrm{D}$, de chromosomes acrocentriques et le groupe $\mathrm{F}$ de chromosomes télocentriques ou subtélocentriques. Les chromosomes $\mathrm{X}$ sont inclus dans le groupe $\mathrm{C}$ (fig. I et 2).

Les bandes des 22 paires de chromosomes se répartissent comme suit :

\section{A. - Groupe A (fig. I)}

Les quatre paires de ce groupe sont submétacentriques ou métacentriques. Elles sont disposées par taille décroissante.

\section{Chromosomes $A_{1}$.}

C'est la plus grande paire du caryotype. Au niveau des bras courts, on compte trois bandes d'égale largeur $(\mathrm{I}-2-3)$. La région du centromère est marquée par une bande sombre (4). Les bras longs présentent deux bandes larges $\left(5^{-6}\right)$.

\section{Chromosomes $A_{2}$.}

Ils sont légèrement plus courts et plus métacentriques que les précédents. Les bras courts montrent trois bandes (I-2-3) ainsi que les bras longs (5-6-7). Une bande sombre existe au niveau du centromère.

\section{Chromosomes $A_{3}$.}

Ils sont nettement submétacentriques. Une bande occupe les bras courts (r) juste au-dessus du centromère. Quatre bandes peuvent être observées au niveau des bras longs. Ces quatre bandes sont groupées deux à deux (2-3 puis 4-5). Plus le chromosome est court, plus elles sont confondues deux à deux. Dans ce cas, les bras longs du chromosome ne présentent que deux bandes sombres.

\section{Chromosomes $A_{4}$.}

Il s'agit de la plus petite paire du groupe. On observe deux bandes au niveau des bras courts (I-2). La bande I terminale est plus étroite que la bande 2 . Trois bandes d'égale largeur sont décelables sur les bras longs (3-4-5).

$$
\text { B. - Groupe } B \text { (fig.I) }
$$

Les quatre paires de ce groupe sont acrocentriques. Elles ont des longueurs voisines, mais sont plus courtes que les précédentes. 

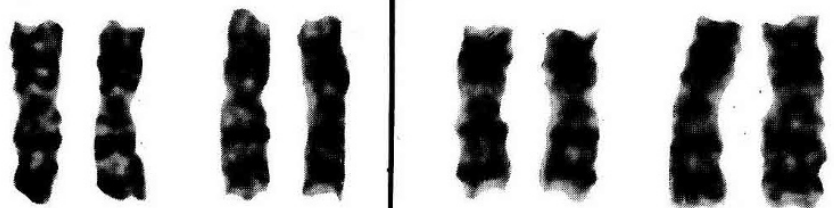

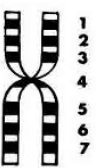
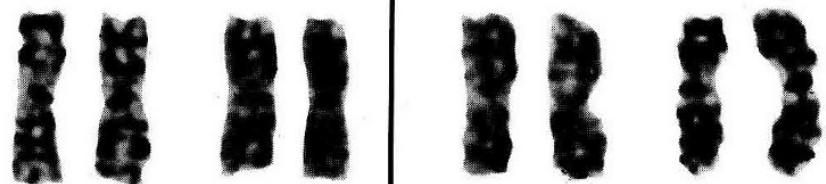

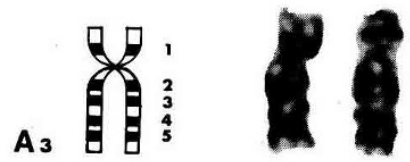
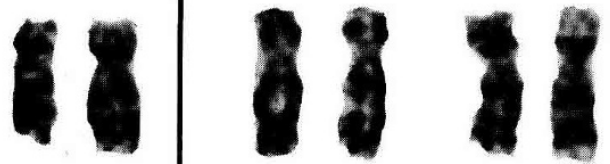

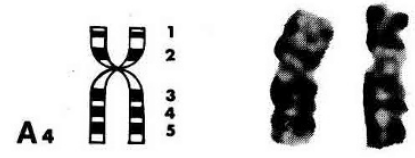
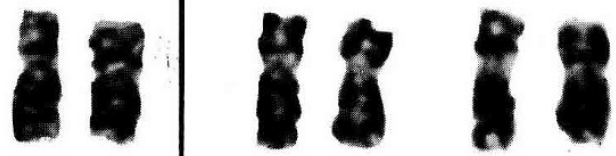

B $6 \square$
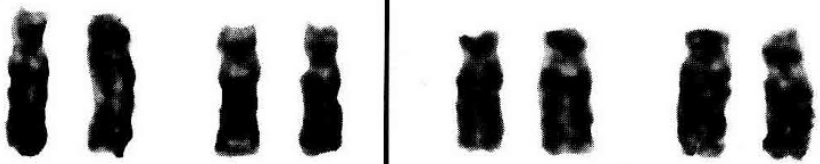

B5 5 E 6
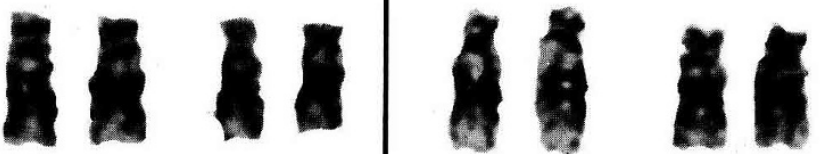

B7 百

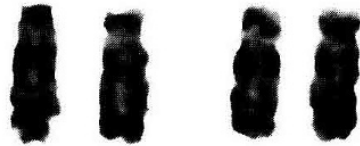

B8 8 G $\begin{array}{lll}1 \\ 3 \\ 3\end{array}$
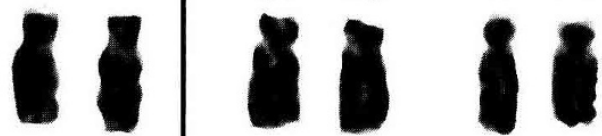

C.
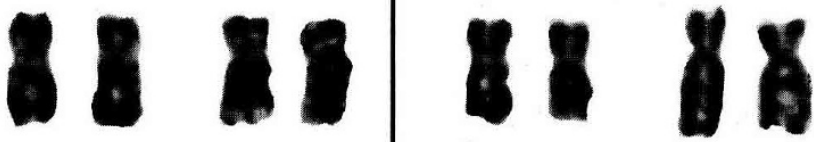

$C_{10} \underbrace{\frac{1}{2}}_{X}$
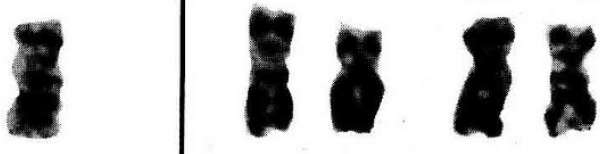

FIG. I. - Le chromosome à bandes du lapin : groupes $A, B$, et $C\left(C_{10}\right)$ 


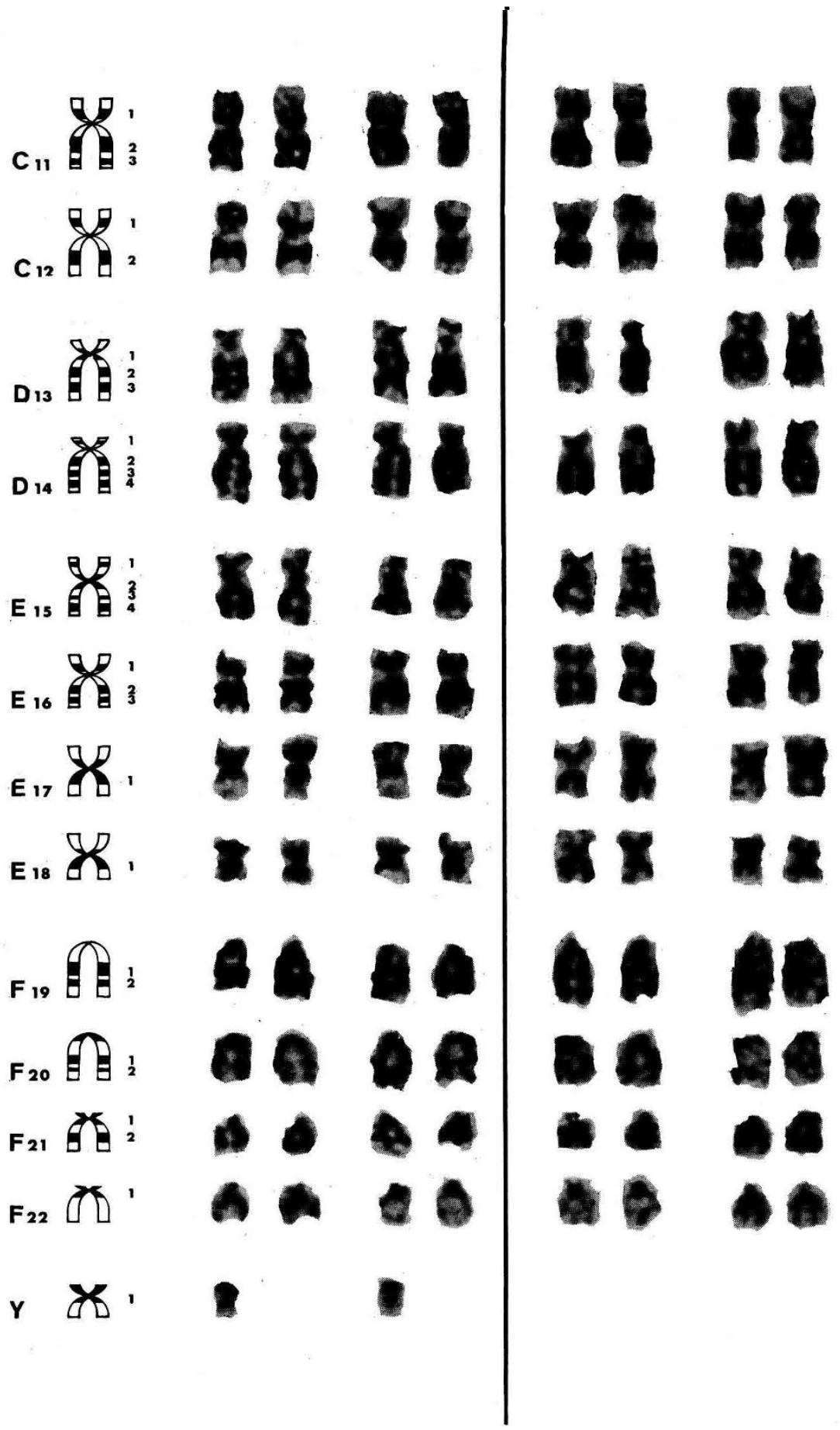

Fig. 2. - Le chromosome à bandes du lapin (suite) : groupes $C\left(C_{11}\right), D, E, F$ et $Y$ 
I. Chromosomes $B_{5}$.

Une bande occupe les bras courts (I). Au-dessous du centromère existe une zone claire, puis quatre à cinq bandes d'égale largeur occupent le reste des bras longs $(2-3-4-5-6)$. Certaines de ces bandes sont souvent confondues.

\section{Chromosomes $B_{6}$.}

Cette paire est pratiquement aussi longue que la précédente, mais ses bandes se répartissent différemment ce qui rend leur distinction aisée. Une bande étroite occupe les bras courts (I). Juste au-dessous du centromère se trouve une bande sombre (2), puis, au milieu des bras longs, on observe deux bandes rapprochées $(3-4)$.

\section{Chromosomes $B_{7}$.}

Leur taille est très voisine de celle des deux paires précédentes. Leurs bandes se répartissent comme suit : une bande sombre au niveau des bras courts (I) ; quatre bandes occupent l'ensemble des bras longs et sont groupées deux à deux (2-3 puis 4-5).

4. Chromosomes $B_{8}$.

Cette paire est nettement plus courte que les trois précédentes. Une bande est située au niveau des bras courts (I). Trois ou quatre bandes d'égale épaisseur se répartissent le long des bras longs $(2-3-4-5)$.

$$
\text { C. - Groupe C (fig. I et 2) }
$$

Ce groupe comporte quatre paires de chromosomes de taille moyenne et à centromère médian ou sub-médian. Une de ces paires est identifiée comme étant les chromosomes $\mathrm{X}$.

\section{Chromosomes $C_{9}$.}

Cette paire est complète chez le mâle. On peut distinguer une bande au niveau de la partie terminale des bras courts (I), et trois bandes d'égale largeur au niveau des bras longs (2-3-4).

\section{Chromosomes $C_{10}$.}

Un seul exemplaire existe chez le mâle tandis que la paire est complète chez la femelle. Il s'agit donc des chromosomes $\mathrm{X}$. La répartition des bandes de ce chromosome le rend facilement reconnaissable. Au niveau des bras courts, on distingue une bande large subterminale (I), puis une bande étroite proche du centromère (2). Une bande nette s'observe au-dessous du centromère (3), les bras longs montrent ensuite un espace clair et se terminent par deux bandes sombres très rapprochées (4-5).

L'identification des chromosomes X du Lapin a toujours été délicate. Les mesures biométriques et les études autoradiographiques ont amené les différents auteurs à définir les chromosomes $\mathrm{X}$ comme une paire de taille moyenne à centromère submédian (Nichols et al., I965; RAY et Wilimiams, I965 ; ISSA et al., I968). Les quatre 
paires du groupe $\mathrm{C}$ peuvent répondre à cette définition. Récemment MARTIN DE LÉON (I972) propose comme principaux caractères cytologiques d'identification d'une part l'hétéropycnose très positive de l'un des $\mathrm{X}$ chez la femelle et d'autre part l'aspect plus fin et moins spiralisé des bras de ce chromosome. Ces caractères sembleraient être ceux du chromosome $\mathrm{X}$ dont la réplication est retardée.

L'existence d'une répartition spécifique des bandes chromosomiques permet de reconnaître sans ambiguité les chromosomes $\mathrm{X}$ chez la femelle comme chez le mâle, même dans le cas assez fréquent où les deux chromosomes $\mathrm{X}$ d'une métaphase apparaissent de taille différente chez la femelle.

\section{Chromosomes $C_{11}$.}

La paire est complète chez le mâle. Les bras courts présentent une bande large juste au-dessus du centromère (I). Au-dessous se trouve une large bande sombre (2), puis au niveau sub-terminal des bras longs existe une dernière bande plus mince (3).

\section{Chromosomes $C_{12}$.}

Cette dernière paire du groupe est aussi complète chez le mâle. Elle montre deux larges bandes caractéristiques l'une au-dessus du centromère $(\mathrm{r})$, l'autre au milieu des bras longs (2). Cette dernière peut parfois se résoudre en deux bandes contiguës.

$$
\text { D. - Groupe D (fig. 2) }
$$

Ce groupe contient deux paires de chromosomes acrocentriques dont la taille est très voisine. La répartition de leurs bandes permet de les distinguer.

\section{Chromosomes $D_{13}$.}

La partie terminale des bras courts est claire, parfois elle montre une fine bande sombre. La région du centromère est occupée par une tache sombre bien visible (I). Deux bandes larges sont situées au milieu des bras longs (2-3).

\section{Chromosomes $D_{14}$.}

Une bande existe au niveau des bras courts (I). Trois, parfois quatre bandes d'égale largeur sont régulièrement réparties sur les bras longs (2-3-4).

$$
\text { E. - Groupe E (fig. 2) }
$$

Quatre paires de petits chromosomes forment ce groupe. Deux paires sont submétacentriques, et les deux dernières métacentriques.

\section{Chromosomes $E_{15}$.}

Il s'agit de la plus grande paire du groupe. Une bande étroite occupe la partie sub-terminale des bras courts $(I)$. La région du centromère est marquée par une nette tache sombre (2). Deux bandes sont visibles sur le reste des bras longs, la première est mince (3), la deuxième sub-distale est plus large (4). 
2. Chromosomes $E_{16}$.

Ils sont légèrement sub-métacentriques. Une bande large est située juste au-dessus du centromère $(\mathrm{I})$, deux bandes larges occupent la plus grande partie des bras longs ( 2 et 3).

3. Chromosomes $E_{17}$.

Une bande sombre s'étale de part et d'autre du centromère, prenant plus d'ampleur du côté considéré comme bras long ( $\mathrm{r}$ ).

4. Chromosomes $E_{18}$.

Ils sont légèrement plus petits que les précédents et présentent la même disposition des bandes. Ces deux paires restent difficiles à distinguer l'une de l'autre.

$$
\text { F. - Groupe } F \text { (fig. 2) }
$$

Deux paires de chromosomes télocentriques et deux paires sub-acrocentriques sont placées dans ce groupe.

\section{Chromosomes $F_{19}$.}

Ils peuvent être caractérisés par deux bandes situées au milieu des bras. La bande I étant plus large que la bande 2 .

\section{Chromosomes $F_{20}$.}

Ils sont légèrement plus petits que les précédents. Une bande étroite est souvent visible au niveau du centromère (I). Deux bandes de largeur différente occupent les bras longs $(2-3)$. Ces bandes sont plus étroites que celles des chromosomes $\mathrm{F}_{19}$.

\section{Chromosomes $F_{21}$.}

Sub-acrocentriques, on observe une bande sombre au niveau du centromère (I), puis une seconde bande au milieu des bras.

\section{Chromosomes $F_{22}$.}

Sub-acrocentriques aussi, leur longueur est voisine de celle de la paire précédente, mais l'aspect caractéristique de leur bande unique ( $\mathrm{r}$ ), localisée au niveau du centromère permet de les distinguer sans ambiguité de la paire $F_{21}$.

$$
\text { G. - Le chromosome } Y \text { (fig. 2) }
$$

Il est facile à reconnaître sur les métaphases mâles. Un peu plus petit que la paire $F_{22}$, son centromère est sub-médian. Une bande sombre occupe la totalité des bras courts et se prolonge sur une courte distance au-delà du centromère.

\section{DISCUSSION}

Bien que les chromosomes du Lapin se répartissent facilement en groupes, il était jusqu'à présent impossible de constituer avec certitude des paires chromosomiques à l'intérieur des groupes. Même la réalisation de l'idiogramme laisse persister 
des incertitudes dans 1'appariement des homologues. En effet, il est fréquent de rencontrer d'une part des inégalités de longueur entre les deux chromosomes d'une même paire et, d'autre part, les chromosomes d'une métaphase donnée peuvent présenter un écart sensible par rapport à la moyenne. Ceci rend très difficile un appariement correct lorsque les chromosomes sont de morphologie très voisine. La liste des paires difficiles à distinguer l'une de l'autre chez le Lapin a été donnée par Gustavsson en I964. La connaissance des bandes chromosomiques de type G de chaque paire d'homologues lève presque toute les ambiguïtés. En effet, la paire $A_{1}$ se distingue de la paire $A_{2}$ par les deux larges bandes de ses bras longs. Il n'est plus possible de confondre entre elles les paires $B_{5}, B_{6}$ et $B_{7}$, chacune ayant ses bandes caractéristiques. A l'intérieur du groupe $C$, chaque paire est identifiée ainsi que les chromosomes $\mathrm{X}$. Les deux paires du groupe $\mathrm{D}$ ne peuvent plus être confondues. Dans le groupe $\mathrm{E}$ trois paires sont aisées à distinguer les unes par rapport aux autres. Les quatre paires du groupe $\mathrm{F}$ sont chacune identifiées.

Trois ambiguïtés cependant persistent encore :

$I^{0}$ Les bandes de la paire $B_{5}$ sont très voisines de celles de la paire $B_{8}$. Deux différences peuvent être notées, d'une part la longueur plus courte du chromosome $\mathbf{B}_{8}$ et d'autre part l'épaisseur souvent plus marquée de la bande 2 du chromosome $B_{5}$.

$2^{\circ}$ Les chromosomes $\mathrm{E}_{18}$ et $\mathrm{E}_{17}$ métacentriques,' de taille voisine et ayant les mêmes bandes restent difficiles à apparier avec certitude.

$3^{\circ}$ Le chromosome $C_{12}$ et le chromosome $E_{16}$ ont une répartition voisine de leurs bandes; mais la longueur du chromosome $\mathrm{C}_{12}$ est nettement plus importante.

Une étude plus détaillée des bandes de ces chromosomes permettra sûrement de les caractériser définitivement. En particulier, elle peut être envisagée en utilisant soit les techniques de bandes doublées d'enregistrement au micro-densitomètre, soit les techniques de microscopie électronique à faible grossissement.

Une bonne connaissance $\mathrm{du}$ caryotype à bandes du lapin est nécessaire pour la détection exacte des chromosomes impliqués dans les cas d'anomalies chromosomiques pathologiques ou non. On sait par ailleurs, que la plupart des avortements spontanés chez 1'Homme et d'autres mammifères sont dus à des anomalies chromosomiques dont la plus fréquente se traduit par une hétéroploïdie des embryons : trisomie d'une paire de chromosome, triploïdie, tétraploïdie (CARR, I97I).

Une étude comparée des bandes chromosomiques de deux espèces voisines telles que le Lièvre $(2 n=48)$ et le Lapin $(2 n=44)$ pourrait permettre d'envisager sous un aspect nouveau l'évolution interspécifique du caryotype de ces deux espèces morphologiquement très voisines.

Reçu pour publication en juillet 1973.

\section{SUMMARY}

\section{G-BAND PATTERN OF RABBIT CHROMOSOMES}

The new cytological techniques developped to obtain the specific banding pattern of each chromosome of a karyotype are now commonly used for cytogenetic studies. One of these tcehniques : proteolytic digestion with trypsin and Giemsa staining (T-G techniqw) has been adjusted 
to rabbit bone marrow cells. The characteristic G-band pattern of the $2 \mathrm{I}$ autosomal pairs is described. $\mathrm{X}$ chromosomes are identified by means of their specific banding. Still some difficulties remain to identify with certainty three pairs of chromosomes having similar lenght, centromeric position and banding pattern. This G-band pattern is reproducible and can be applied in the study of chromosome abnormalities.

\section{RÉFÉRENCES BIBLIOGRAPHIQUES}

Berger R., 1972. Étude du caryotype du Porc avec une nouvelle technique. Expt. Cell. Res. 75, 298-300.

CARr D. H., I97I. Genetic basis of abortion. Ann. Rev. Gen., 5, 65-80.

Dave M. J., Takagi N., Orshi H., KIKUChI Y., r965. Chromosome studies on the Hare and the Rabbit. Proc. Jap. Acad., 41, 244-248.

Echard G., I973. Étude des bandes chromosomiques du porc et de trois différentes souches de rein de porc en culture. (PKI5, F et RP). Ann. Génét. Sel. Anim., 5, I, I-2r.

Feichiermer N. S., 1972. Chromosome abnormalites and embryo death in farm animals. Vet. Rec., 90, 24I-243.

Gustavsson I., I964. Somatic chromosomes of the Rabbit. Acta Vet. Scand., 5, 362-369.

Gustavsson I, HAGEltorn M., Johanson C, ZECH L., I972. Identification of the pig chromosomes by the quinacrine mustard fluorescence technique. Expt. Cell. Res., 70, 471-474.

HANSEN K. M., I972. Bovine chromosomes identified by quinacrine mustard and fluorescence microscopy. Hereditas, 70, 225-234.

HANSEN K. M., I972. The karyotype of the pig (Sus scrofa domestica) identified by quinacrine mustard staining and fluorescence microscopy. Cytogenetics, 11, 286-294.

HANSEN K. M., I973. Heterochromatin (C-Bands) in bovine chromosomes. Hereditas, 73, 65-70.

HANSEN K. M., I973. Identification of goat chromosomes by the Quinacrine Mustard Fluorescence Technique. Hereditas, 73, 152.

Hofsaess F. R., Meacham T. N., I97I. Chromosome abnormalities of early Rabbit embryos. J. Exp. Zool., 177, 9-12.

Issa M., Atherton G. W., Blank C. E., I968. The chromosomes of the domestic Rabbit : Oryctolagus cuniculus. Cytogenetics, 7, 36r-375.

LEe C. L. Y., Welch J. P., Winsor E. J. T., I972. Banding patterns in human chromosomes : production by proteolytic enzymes. $J$. of Heredity, 63, 296-297.

MARTIN DE LÉON P. A., I972. Morphologically distinctive $\times$ chromosome in the female complement of the rabbit. Canad.J. Genet. Cytol., 14, 8I7-82I.

Melander Y., 1956. The chromosome complement of the rabbit. Hereditas, 42, 432-435.

Nadler C F, Hoffmann R. S., Woolf A, r973. G-band Patterns as chromosomal markers, and the interpretation of chromosomal evolution in wild sheep (Ovis). Experientia, 15, II7-IIg.

Nichols W. W., Levan A., Hansen-Melander E., Melander Y., 1965. The Idiogram of the Rabbit. Heveditas, 53, 63-76.

Painter T. S, 1926. Studies in mammalian spermatogenesis. IV. - The chromosomes of the Rabbit. J. Morphol, 43, r-43.

Paris Conference, I97I-I972. Standardization in Human cytogenetics Cytogenetics, 11, 3I7-359.

SeABright M, r97I. A rapid banding technique for human chromosomes. Lancet, 11, 971-972.

WANG H. C., FEDORofF S., I972. Banding in human chromosomes treated with trypsin. Nature (New Biology), 235, 52-54.

Williams T. W., RAY M., 1965. A method for culturing leucocytes of Rats and Rabbits. Cytogenetics, 4, 365-368. 